\title{
Boron doped nanocrystalline diamond temperature regulator for sensing applications
}

\author{
Tim Clukers ${ }^{*, 1,2}$, Bart Van Grinsven ${ }^{1}$, Thijs Vandenryt ${ }^{1,2}$, Stoffel D. Janssens ${ }^{1}$, Patrick Wagner ${ }^{1,3}$, \\ Ward De Ceuninck ${ }^{1,3}$, Ronald Thoelen ${ }^{1,2}$, Michaël Daenen ${ }^{*, 1,2}$, and Ken Haenen ${ }^{* *, 1,3}$ \\ ${ }^{1}$ Hasselt University, Institute for Materials Research (IMO), Wetenschapspark 1, 3590 Diepenbeek, Belgium
${ }^{2}$ Xios University College Limburg, Agoralaan H, 3590 Diepenbeek, Belgium
${ }^{3}$ IMEC vzw, Division IMOMEC, Wetenschapspark 1, 3590 Diepenbeek, Belgium
}

Received 3 May 2010, revised 14 July 2010, accepted 15 July 2010

Published online 13 August 2010

Keywords B-NCD-layer, PID-control, temperature regulator

*Corresponding author: e-mail tim.clukers@xios.be; michael.daenen@xios.be, Phone: +32 113707 77, Fax: +32 11 360780

**e-mail ken.haenen@uhasselt.be

In search for a better way to monitor the hybridization and denaturation of DNA onto a diamond based sensor, precise knowledge about the conditions of the immediate surroundings is very critical. One of the factors that have a great influence on the stability of the measurements is the temperature of the liquid environment in which these measurements take place. With this as a focal point, the design of a precise temperature regulator based on a boron doped diamond thin film is a key factor to achieve accurate measurements on a standalone basis. In this work temperature control is achieved making use of a thin boron doped nanocrystalline diamond (B-NCD) film, which, in combination with a proportional-integral-derivative-control (PID), is able to maintain a stable temperature with an accuracy better than $0.1{ }^{\circ} \mathrm{C}$. By letting the B-NCD-layer act as a resistor together with the appropriate control it is possible to maintain a stable temperature in a range going from room temperature till $70^{\circ} \mathrm{C}$, with an accuracy exceeding a temperature variation $0.1^{\circ} \mathrm{C}$. The first prototype makes use of a reference temperature sensor, to verify the accuracy of the results.

\section{Introduction}

1.1 General principle If one wants to monitor the hybridization and denaturation of DNA, it is of great importance that the conditions of the immediate surroundings are known exactly. When working with nanocrystalline diamond (NCD) surfaces to tether DNA onto these preliminary conditions are in no way different [1]. The stability of such measurements depends greatly on the temperature of the surrounding liquid. The hybridization and denaturation of DNA is a balanced reaction that starts at $37^{\circ} \mathrm{C}$ and continues up to $70^{\circ} \mathrm{C}$, but each working temperature has a different time constant. Therefore a decent DNA-denaturation/hybridisation experiment can only be achieved at a highly stable temperature [2]. As diamond can be made semiconducting, while at the same time providing a very stable platform for the attachment of biological entities like DNA, boron doped nanocrystalline diamond (B-NCD) films are an ideal candidate material to use as a temperature regulator and temperature sensor [3-6]. The conductivity of such layers can be controlled over nearly 10 orders of magnitude by a simple variation of the boron content [7]. As B-NCD is a semi-conducting material, decreasing temperature will decrease the amount of charge carriers and the resistivity rises accordingly, and by this principle the resistivity of the B-NCD-layer can be directly linked to the temperature of its immediate surroundings [8]. At the same time, by letting the B-NCD-layer act as a resistor, it is possible to use the Joule effect to heat these surroundings, providing an elegant method to combine the function of heating element and temperature sensor in one device.

1.2 Measurement setup A $150 \mathrm{~nm}$ thick B-NCDlayer was grown onto a quarts substrate of $1 \times 1 \mathrm{~cm}^{2}$ by the chemical vapour deposition (CVD) method using an ASTeX 6500 reactor, after a pre-treatment step with a colloidal solution of ultra dispersed nanodiamond [5, 9], and a methane concentration of $2.5 \%$ with respect to the hydrogen gas content. During the deposition $5000 \mathrm{ppm}$ thrimethyl 
boron-to-methane ratio in the gaseous phase was used and an overall deposition temperature of $700{ }^{\circ} \mathrm{C}$ was maintained as measured with a two colour pyrometer. The number of charge carriers and carrier mobility at room temperature (RT) are respectively $2.1 \times 10^{21} / \mathrm{cm}^{3}$ and $0.65 \mathrm{~cm}^{2} / \mathrm{V} \mathrm{s}$. These data are in perfect accordance with the work of Gajewski et al., where the Mott transition was proven to lie between 2 and $3 \times 10^{20} / \mathrm{cm}^{3}$ for heavily B-doped NCD films [7]. Hence, metallic behaviour of the film under study can be expected, which is reflected in the specific resistivity of the grown sample at RT, being $6 \times 10^{-5} \Omega \cdot \mathrm{m}$ while the sample has a side to side resistance of $450 \Omega$, as measured from the centre of one side to the centre of the opposite side. To remove the surface conductivity related to the hydrogen termination of the surface ozone plasma was employed, leading to oxygen groups at the surface. For insuring the highest accuracy of the electrical measurements, two Ti/Al contact strips with a width of $1 \mathrm{~mm}$ were sputtered over the whole length of the B-NCD sample using a homemade sputtering installation. During the calibration carried out to investigate the relation between the temperature and the resistance of the layer, the temperature regulation and measurement of the sample was carried out by a SÜSS semiautomatic wafer prober system PA200 using four-contact needles connected to a HP3458A. The corresponding resistance was measured by a four-point measurement using a current of $1 \mathrm{~mA}$. After calibration a newly designed measurement cell was developed to carry out the temperature regulation of the actual thermistor. The measurement cell, as shown in Fig. 1, consists out of a plexiglas body with a plexiglas cover pressing down the B-NCD sample. The diamond layer is facing downwards and is pressed onto the rubber gasket avoiding possible leakages when measurements are carried out in a fluidic environment. The two integrated golden spring contacts insure a good electric contact with the two contact strips of the sample. The bottom side of the temperature regulator is closed with a quarts glass cover providing an optical pathway that can be used for

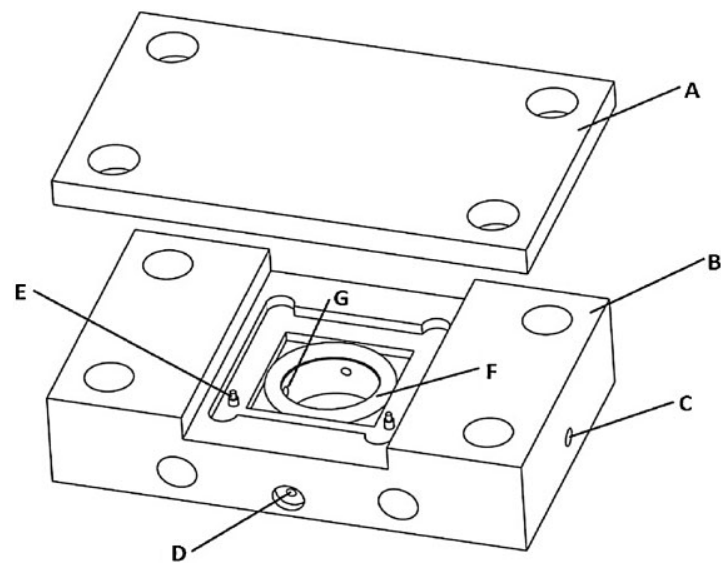

Figure 1 Thermistor cell: (A) plexiglas cover, (B) plexiglas base, (C) fluid inlet, (D) thermocouple feed through, (E) golden contact spring, $(\mathrm{F})$ rubber gasket, $(\mathrm{G})$ fluid outlet. simultaneous measurements such as fluorescence microscopy. The testing cell is filled with distilled water with a resistivity of $18 \mathrm{M} \Omega \cdot \mathrm{cm}$ and heated to a pre-defined set point temperature. As feedback loop a thermocouple is integrated to carry out an accurate temperature measurement. The control settings for the PID-control of the temperature regulator cell were carried out by an application specified LabVIEW program. After measuring the latent time of induced temperature step response, the PID settings were calculated using the Ziegler/Nichols method [10]. The necessary measurements, current and corresponding voltage, were done by a four-point measurement connected to the golden spring contacts using a Keithley 2400 Source meter coupled via a general purpose interface bus to the computer running the dedicated LabVIEW program.

\section{Results}

2.1 Temperature dependent resistance As proof of principle a series of calibration measurements were carried out by independently heating the B-NCD sample in steps of $10{ }^{\circ} \mathrm{C}$ with the wafer prober system while measuring the resistance. Measurements were taken with a sampling frequency of $10 \mathrm{~Hz}$ over a period of $20 \mathrm{~min}$ to make sure that the temperature was stable and the measurements of the resistance were as accurate as possible, displaying a maximum standard deviation of under $0.0016 \%$. The resistance measurements were averaged, according to matching set point temperature as a reference, to create a fit to a linear curve. As shown in Fig. 2 the resistance of the BNCD sample drops accordingly to the rise of the temperature.

In Fig. 3 the resistance is plotted as function of temperature. Although only a small change in resistance is expected for metallic type conductive films in the used temperature window [7], it is obvious that a linear decrease can be observed. The fit equation, as can be seen in the data frame in Fig. 3, states that the linear fit of the measurements proceeds according to the equation $Y=-0.326 x+453.6$. The corresponding errors are 0.00172 on the value of

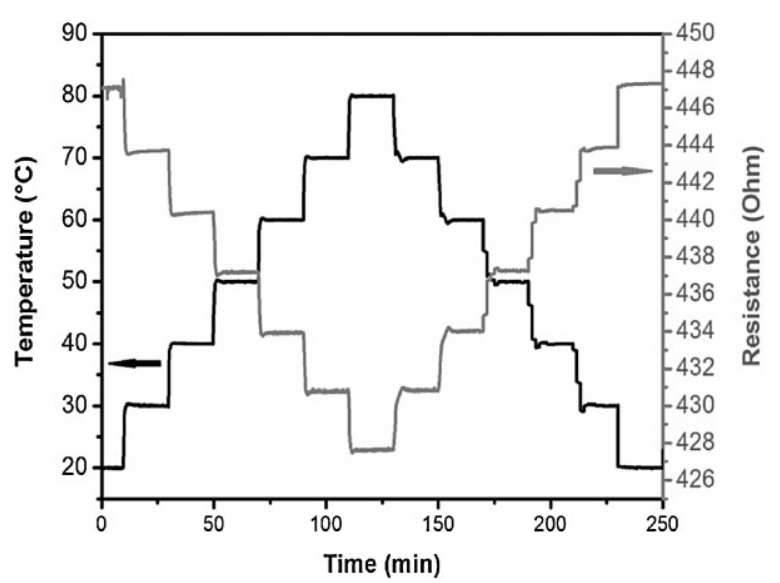

Figure 2 Calibration measurement showing resistance decrement/ increment with rising/declining temperature. 


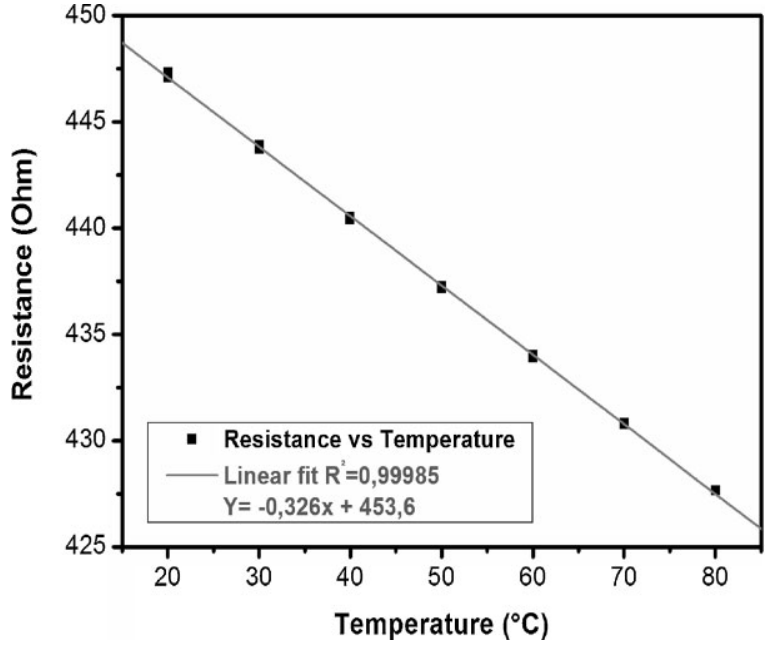

Figure 3 Linear fit of the resistance in function of the temperature.

$A=0.326$ and 0.08808 on the value of $B=453.4$ while the coefficient of determination $\left(R^{2}\right)$ is 0.99 . This shows that the resistance drop of the B-NCD under influence of the rising temperature is practically linear [11]. The error bars on the measurements are not included in the graph because the standard deviation on the measurements is smaller than the dots of the measurement points in the graph itself.

2.2 Determining PID settings Before any feedback control system can be implemented, the step response for the heating of the B-NCD film must be measured. In this stage the actual temperature regulator cell was used. Measuring the step response was done by sending a constant current of $30 \mathrm{~mA}$ through the B-NCD sample. The key factor to determine the $\mathrm{P}, \mathrm{I}$ and $\mathrm{D}$ values of the steering is the latent time of the temperature increment. The latent time of a feedback control system is the delay time between the start of the steering signal and the actual response of the controlled system. This latent time was derived from measurements of the step response. The temperature curve in Fig. 4 is the actual curve from which the latent time, and afterwards the PID settings, were derived, with the former being $7.83 \mathrm{~s}$. After calculations, using the Ziegler/Nichols method, the derived values for the control were $P=0.024$, $I=15.68$ and $D=3.92$. While measuring the temperature increment the voltage decrement was monitored. As proven in the calibration, the resistance dropped according to the rising temperature. Because of the direct proportionality of the relation between the voltage and the resistance, when using a constant current, the value for the conductivity can be directly derived from the measured voltage of the B-NCD sample. In Fig. 4 the increase of conductivity due to the increase of temperature is plotted as a function of the time.

2.3 PID controlled diamond surface temperature regulator With the obtained parameters from the step response (see Fig. 4), the PID-control was implemented

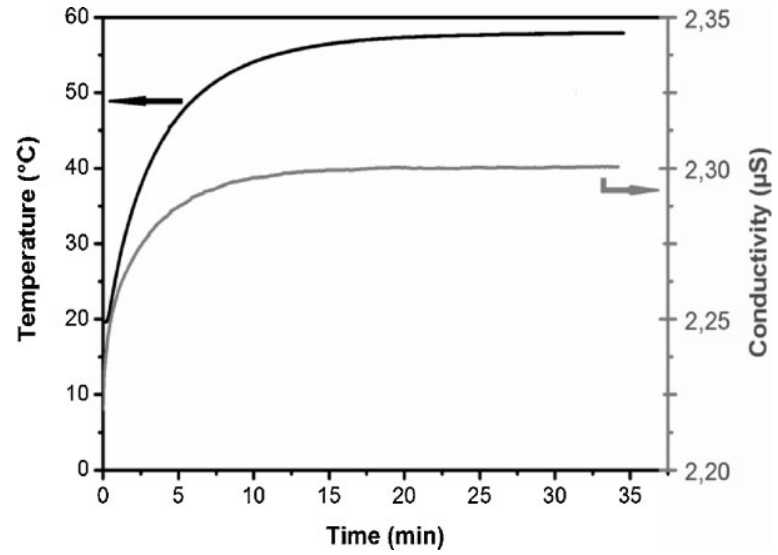

Figure 4 Conductivity and temperature of the B-NCD sample as function of time for a constant current of $30 \mathrm{~mA}$ passed through the sample.

onto the temperature regulator cell. This control was carried out by a homemade LabVIEW program and the goal was to enable an automated temperature control exceeding an accuracy of $0.2{ }^{\circ} \mathrm{C}$ varying around the set point temperature. After a first test with a set point of $37^{\circ} \mathrm{C}$ a temperature overshoot till $37.04{ }^{\circ} \mathrm{C}$ was measured and subsequently a stable temperature was achieved with a maximum variation of $0.02{ }^{\circ} \mathrm{C}$. After the cooling down to RT a second test was carried out with $70^{\circ} \mathrm{C}$ as a set point. With the latter, the temperature overshoot decreased to $70.03{ }^{\circ} \mathrm{C}$. After the overshoot the temperature remained stable with a maximum variation of $0.01{ }^{\circ} \mathrm{C}$. This difference of the maximum variation of the temperature can be explained by the fact that at a higher temperature more heat is dissipated. When there is more energy dissipation the tendency to overheat will be much smaller and by this it will be easier to control the temperature more accurately. The control signal can then be more constant, avoiding unnecessary fluctuations to keep a stable temperature. After the primary measurements, a sequence of set points were programmed to test the time response needed to go to an elevated temperature and back. As can be seen in Fig. 5 the temperature is varied between 60 and $37^{\circ} \mathrm{C}$. After $13 \mathrm{~min}$ the time to go back to RT was tested followed by another rise to $37^{\circ} \mathrm{C}$.

The temperature increment from $37^{\circ} \mathrm{C}$ to a stable $60^{\circ} \mathrm{C}$ was accomplished within a peak time of $113 \mathrm{~s}$ accompanied with an overshoot not exceeding $0.03{ }^{\circ} \mathrm{C}$. The increment time to the set point temperature only took $47 \mathrm{~s}$. The temperature measured reached its set point value within a settling time of $127 \mathrm{~s}$, with an accuracy of $0.01^{\circ} \mathrm{C}$. These data clarify that future measurements involving DNA hybridization and dehybridization can be done within minutes, and at a higher accuracy and a more stable temperature then formerly possible [12]. The fact that this setup is able to maintain such a stable temperature at a standalone basis proves that this thermistor is a valid solution for highly accurate temperature based measurements. 


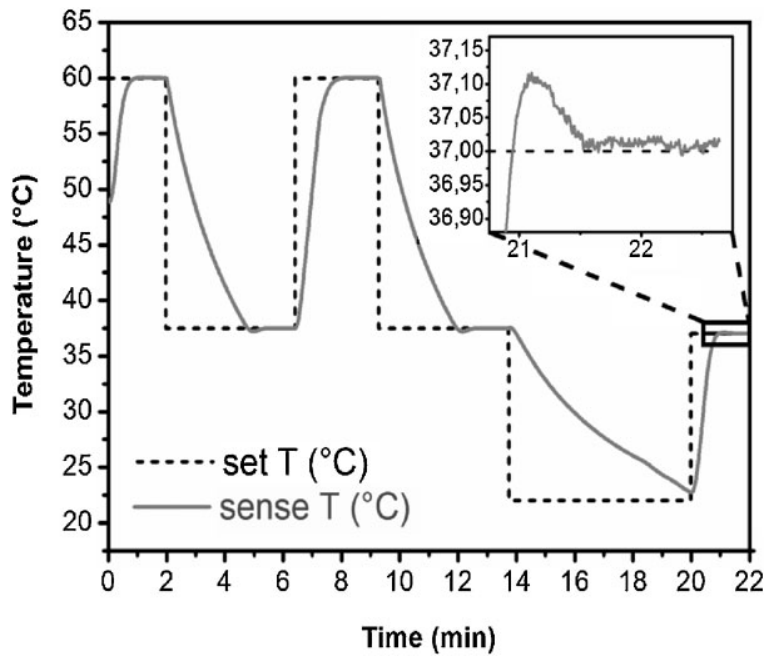

Figure 5 Sequence of set point temperatures controlled by a PIDcontrol. The inset shows the overshoot and regulation of the last step to $37^{\circ} \mathrm{C}$.

3 Conclusion The design of an accurate thermistor based on a boron doped nanocrystalline diamond thin film proved to perform highly stable and accurate measurements. The designed stand alone device enables a temperature increment to a given set point temperature within less than 2 min, maintaining such temperatures with great stability and an accuracy exceeding $0.01{ }^{\circ} \mathrm{C}$. The duality of the setup is found in the fact that it works as a thermistor to control and maintain an environmental fluid on the exact set point temperature, while possibly serving as a stable platform to bind molecules such as DNA on its surface. By letting the B-NCD layer act as a resistor, due to its semi-conducting capacities, together with the appropriate PID control program it is possible to maintain a stable temperature in ambient circumstances. While this prototype still has a reference temperature sensing thermocouple, additional calibration steps will also allow the thermistor to be used as a temperature measurement device. The field of usage can even be extended to any temperature regulated measurement that needs to be executed in chemically and physically harsh environments.

Acknowledgements This work was financially supported by the Research Programs G.0068.07 and G.0430.07 of the Research Foundation - Flanders (FWO), the Methusalem 'NANO network Antwerp - Hasselt', and the IAP-P6/42 project 'Quantum Effects in Clusters and Nanowires'. We gratefully acknowledge the work of J. Soogen, J. Mertens, G. Lekens and J. Baccus.

\section{References}

[1] B. van Grinsven, T. Vandenryt, S. Duchateau, A. Gaulke, L. Grieten, R. Thoelen, S. Ingebrandt, W. De Ceuninck, and P. Wagner, Phys. Status Solidi A 207(4), 919-923 (2010).

[2] V. Vermeeren, N. Bijnens, S. Wenmackers, M. Daenen, K. Haenen, O. A. Williams, M. Ameloot, M. vandeVen, P. Wagner, and L. Michiels, Langmuir 23, 13193-13202 (2007).

[3] W. Yang, O. Auciello, J. E. Butler, W. Cai, J. A. Carlisle, J. E. Gerbi, D. M. Gruen, T. Knickerbocker, T. L. Lasseter, J. N. Russell, Jr., L. M. Smith, and R. J. Hamers, Nature Mater. 1, 253-257 (2002).

[4] P. Christiaans, V. Vermeeren, S. Wenmackers, M. Daenen, K. Haenen, M. Nesladek, M. vandeVen, M. Ameloot, L. Michiels, and P. Wagner, Biosens. Bioelectron. 22, 170-177 (2006).

[5] M. Daenen, O. A. Williams, J. D'Haen, K. Haenen, and M. Nésladek, Phys. Status Solidi A 203(12), 3005-3010 (2006).

[6] M. Werner, V. Schlichting, and E. Obermeier, Diamond Relat. Mater. 1, 669-672 (1992).

[7] W. Gajewski, P. Achatz, O. A. Williams, K. Haenen, E. Bustarret, M. Stutzmann, and J. A. Garrido, Phys. Rev. B 79, 045206 (2009).

[8] J. P. Bade, S. R. Sahaida, B. R. Stoner, J. A. von Windheim, J. T. Glass, K. Miyata, K. Nishimura, and K. Kobashi, Diamond Relat. Mater. 2, 816-819 (1993).

[9] O. A. Williams, O. Douhéret, M. Daenen, K. Haenen, E. Osawa, and M. Takahashi, Chem. Phys. Lett. 445(4-6), 255-258 (2007).

[10] V. J. VanDoren, Control Eng. 50(7), 30-32 (2003).

[11] S. Grimnes and O. G. Martinsen, Bioimpedance and Bioelectricity - Basics (Academic Press, San Diego, 2000).

[12] M. N. H. Z. Alam, D. Schäpper, and K. V. Gernaey, J. Micromech. Microeng. 20, 055014 (2010). 